\title{
Modelo alternativo de tratamento de vulvovaginite causada por Candida glabrata com uma fração de $n$-butanol das folhas de Terminalia catappa
}

\author{
Alternative treatment of vulvovaginitis caused by Candida glabrata with a fraction of \\ n-butanol from leaves of Terminalia catappa
}

\begin{abstract}
Carlos Henrique Lopes Rocha1; Flaviane Maria Galvão Rocha1; Cristina de Andrade Monteiro ${ }^{2}$
\end{abstract}

Resumo: O objetivo principal deste trabalho foi avaliar o perfil de susceptibilidade de isolados clínicos de Candida glabrata através da técnica de difusão em ágar a uma fração extraída com $n$-butanol das folhas de Terminalia catappa (F-OHf), comparando este tratamento com um outro realizado com o antifúngico de uso clínico e tradicional, o fluconazol (FCZ). A F-OHf apresentou atividade inibitória contra os isolados de C. glabrata, as zonas de inibição de crescimento variaram entre 19 a $27 \mathrm{~mm}$ para o tratamento com $40 \mathrm{mg} / \mathrm{mL}$ e entre 24 a $31 \mathrm{~mm}$ para a concentração $100 \mathrm{mg} / \mathrm{mL}$, para o $\mathrm{FCZ}$ as zonas de inibição variaram de 0 a $5 \mathrm{~mm}$. Em sua totalidade, F-OHf pode ser uma alternativa promissora para o tratamento da candidíase, estes resultados representam novas perspectivas para futuras pesquisas em continuação a este estudo para desenvolver terapias alternativas no combate destas infecções.

Palavras-chave: Candida glabrata, fluconazol, Terminalia catappa

\begin{abstract}
The main objective of this work was to evaluate the susceptibility profile of Candida glabrata clinical isolates through the agar diffusion technique to a fraction extracted with $n$-butanol from the leaves of Terminalia catappa (F-OHf), comparing this treatment with another performed with the traditional and traditional antifungal, fluconazole (FCZ). F-OHf showed inhibitory activity against C. glabrata isolates, growth inhibition zones ranged from 19 to $27 \mathrm{~mm}$ for the $40 \mathrm{mg} / \mathrm{mL}$ treatment and 24 to $31 \mathrm{~mm}$ for the $100 \mathrm{mg} / \mathrm{mL}$ for the FCZ the zones of inhibition ranged from 0 to $5 \mathrm{~mm}$. In all, F-OHf may be a promising alternative for the treatment of candidiasis, these results represent new perspectives for future research in continuation of this study to develop alternative therapies in the fight against these infections.
\end{abstract}

Key words: Candida glabrata, fluconazole, Terminalia catappa

\footnotetext{
${ }_{1}^{1}$ Mestre em biologia Parasitária- laboratório de Microbiologia aplacada da Universidade CEUMA -

Campus Renascença. Universidade CEUMA, Maranhão

2 Docente Permanente do Programa de Mestrado em Biologia Parasitária da Universidade CEUMA -

Campus Renascença. Universidade CEUMA, Maranhão
} 


\section{Introdução}

As infecções fúngicas crescentes representam um problema grave de Saúde pública ${ }^{1}$, Candida albicans uma levedura muito comum nessas situações é, geralmente a espécie mais frequentemente isolada ${ }^{2,3}$. Outras espécies de Candida não albicans também são reportadas causando infecções sistêmicas, superficiais e vulvovaginais ${ }^{4,5}$.

Um estudo epidemiológico demonstrou que $95 \%$ dos casos de candidemia são causadas por cinco espécies de Candida, no entanto, a maior frequência é de Candida albicans, seguida por C. parapsilosis, C. glabrata, C. tropicalis e C. krusei $6,7,8,9$. C. glabrata geralmente é a terceira ou em alguns casos a quarta em relação a frequência de isolamento em pacientes com vulvovaginite ${ }^{2,10}$.

C. glabrata é um microrganismo comensal em superfícies de mucosas, podendo ser portanto, uma das causas da vulvovaginite, infectando tanto indivíduos saudáveis quanto debilitados ${ }^{11}$. As opções terapêuticas usadas no combate as infecções causadas pelas espécies de Candida são limitadas, sendo resumida em três classes de compostos: os azólicos, polienos e as equinocandinas, Anfotericina B é um polieno, que apesar da sua eficiência, apresenta alta toxicidade ${ }^{12}$.

A prescrição mais comum em casos de candidíase vulvovaginal é o azólico fluconazol, este atualmente tem seu uso limitado pela resistência apresentada por algumas espécies do gênero Candida ${ }^{15,16}$. Muitos trabalhos mostram que a espécie $C$. glabrata apresenta resistência intrínseca ao fluconazol ${ }^{17,18}$. Nesse contexto, modelos alternativos de tratamentos são necessários, pois 0 desenvolvimento de medicamentos antifúngicos é relativamente mais lento em comparação com os antibacterianos e a resistência às drogas, o que reduz muito a eficácia dos antifúngicos conhecidos tornando a busca por novas estratégias um mecanismo viável para novos candidatos $^{19}$.

Esses relatos impulsionam a pesquisa a base de plantas medicinais. Compostos isolados a partir destas são utilizados por apresentarem propriedades antimicrobianas empiricamente 20 . Terminalia catappa, surge dessa forma como promissora no tratamento das infecções causadas pelas espécies de Candida ${ }^{21}$. Terminalia catappa é uma planta pertencente à família Combretaceae, nativa do sudeste da África ${ }^{22}$. A atividade microbiana dos extratos de T. catappa é bastante divulgada, o extrato clorofórmico e etanólico das suas folhas apresentam atividade contra várias microrganismos, incluindo leveduras da espécie Candida, Bacillus megaterium, Citrobacter freundii, Micrococcus flavus, Alcaligenes faecalis, Enterobacter aerogenes, Salmonella typhimurium, Klebsiella pneumoniae, Escherichia coli, Streptococcus faecalis, Streptococcus cremoris, Streptococcus agalactiae, e Candida tropicalis. Além disso, baixas concentrações dos extratos de diversas partes da planta são necessárias para inibir o desenvolvimento microbiano ${ }^{22}$.

Vários fitoconstituintes como flavonoides, carotenoides e fenóis estão presentes, e pode ser atribuído a eles a excelente atividade contra microrganismos patogênicos ${ }^{23}$.

Dessa forma o objetivo principal deste trabalho é avaliar o perfil de susceptibilidade de isolados clínicos de Candida glabrata através da 
técnica de difusão em ágar a uma fração extraída com $n$-butanol das folhas de $T$. Catappa (F-OHf), comparando este tratamento com um outro realizado com o antifúngico de uso clínico e tradicional, o fluconazol (FCZ).

\section{Material e Métodos}

\section{Amostras de Candida glabrata}

Os isolados clínicos da espécie Candida glabrata CgCV19; CgCV10; CgCV10 e CgCV4 foram utilizados neste estudo são provenientes de amostras clínicas obtidas a partir de secreção vaginal de pacientes com candidíase e assintomáticas, cedidas gentilmente do Laboratório de Micologia Médica da Universidade Ceuma São Luís - MA (aprovado pelo comitê de ética em pesquisa da Universidade CEUMA, №: 813.402/2014). Os isolados foram previamente identificados pelo sistema automatizado VITEK (BioMérieux), foi preparado um estoque contendo os isolados mantidas a $-20^{\circ} \mathrm{C}$ em caldo $\mathrm{BHI}$ (Brain Heart Infusion- Acumedia Manufactures) para posterior uso.

\section{Preparo do inóculo}

Subcultivos foram realizados em Ágar Sabouraud Dextrose (ASD) por $48 \mathrm{~h}$ a $37^{\circ} \mathrm{C}$. O inóculo foi preparado em solução salina esterilizada $(\mathrm{NaCl}$ $0,85 \%$ ) e a contagem de células ( $1 \mathrm{a}$ $5 \times 10^{6} \mathrm{UFC} / \mathrm{mL}$ ) foi realizada em hemacitômetro (Câmara de Neubauer).

Obtenção da fração de $n$-butanol das folhas de $T$. catappa
Inicialmente as folhas de $T$. catappa foram coletadas e suas superfícies foram limpas com álcool $70 \%$ e secas com papel toalha para evitar proliferação de fungos do ambiente. Essas folhas foram colocadas a temperatura ambiente $e$ posteriormente foram trituradas com auxílio de um triturador mecânico. Passagens sucessivas do pó obtido foram feitas em solventes, obedecendo uma ordem crescente de polaridade. Foram utilizados os solventes hexano, clorofórmio, acetado de etila e $n$-butanol (Merck, Darmstadt, Germany). A fração nbutanol foi acondicionada a $0^{\circ} \mathrm{C}$ para realização do teste de Ágar difusão.

\section{Testes de susceptibilidade antifúngica através do teste de Ágar Difusão}

Os testes de difusão em Ágar foram feitos de acordo com 0 protocolo descrito no documento M44A2 do Clinical And Laboratory Standards Institute ${ }^{24}$, com algumas adaptações. O teste consiste em semear o microrganismo em placas de Petri contendo o meio de cultura ASD. Em determinados pontos desse meio foram feitos orifícios e adicionadas alíquotas de $40 \mu \mathrm{L}$ das substâncias testadas. Após o tempo de incubação a cultura é avaliada. Quando as substâncias testadas exercem ação inibitória no crescimento dos microrganismos cultivados, uma região sem crescimento visível é formada, a qual é determinada pelo diâmetro do halo de inibição formado no meio de cultura. A ação inibitória é diretamente proporcional ao diâmetro do halo.

A zona de inibição do crescimento (ZIC) foi mensurada e o 
cálculo foi realizado para obtenção dos valores para plotagem dos gráficos. O cálculo consistiu em subtrair do diâmetro da zona de inibição total menos o diâmetro do poço perfurado onde foram colocadas as substâncias como ilustrado na Figura 1, dos resultados foi estabelecida uma média. Para a interpretação dos resultados do teste de difusão contra os isolados, utilizouse o documento M44-A2 do Clinical And Laboratory Standards Institute ${ }^{24}$.

O antifúngico fluconazol, utilizado como controle positivo (64 $\mu \mathrm{g} / \mathrm{mL}$ ) e a fração de $n$-butanol das folhas de $T$. catappa $(100 \mathrm{mg} / \mathrm{mL}$ e 20 $\mathrm{mg} / \mathrm{mL}$ ), foram aplicados nos poços feitos no ágar e incubados a $37^{\circ} \mathrm{C}$. Um controle negativo foi realizado com PBS 1x (phosphate-buffered saline, $\mathrm{pH} 7,2)$. Após 24 h, o halo de inibição do crescimento foi medido Foi realizada nova leitura após $48 \mathrm{~h}$ de incubação quando o crescimento foi insuficiente para a leitura até $24 \mathrm{~h}$.

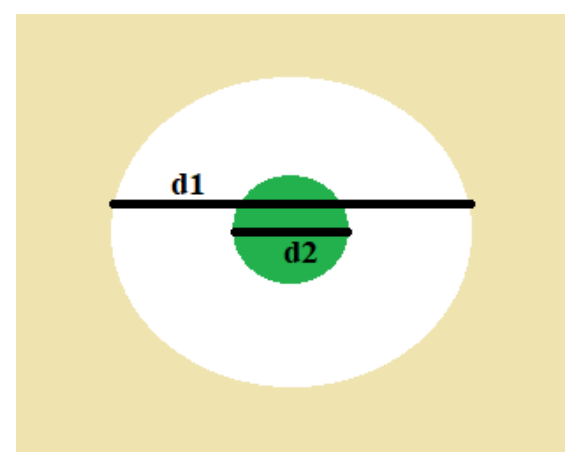

Figura 1. Mensuração da zona de inibição das substâncias testadas pelo método ágar difusão através da fórmula $\mathrm{ZIC}=\mathrm{d} 1-\mathrm{d} 2$ onde: $\mathrm{ZIC}=$ zona de inibição do crescimento; $\mathrm{d} 1=$ diâmetro da zona de inibição + poço; d2= diâmetro do poço onde foram colocada as substâncias.

\section{Análise estatística dos dados}

Os resultados foram analisados estatisticamente quanto às diferenças significativas entre os grupos, por meio da análise de variância (ANOVA) e o teste de múltiplas comparações de Bonferroni. O nível de significância de $95 \%$ foi considerado para que as medidas fossem significativamente diferentes $(p<0,05)$.

\section{Resultados}

Os resultados obtidos demonstraram que a $\mathrm{F}-\mathrm{OHf}$ apresenta atividade inibitória contra os isolados de C. glabrata, as zonas de inibição de crescimento para esses isolados variaram entre 19 a $27 \mathrm{~mm}$ para o tratamento com $40 \mathrm{mg} / \mathrm{mL}$ e entre 24 a $31 \mathrm{~mm}$ para a concentração 100 $\mathrm{mg} / \mathrm{mL}$ (Figura 2). Em contradição, a atividade inibitória de fluconazol não foi satisfatória, as zonas de inibição de crescimento variaram de 0 a $5 \mathrm{~mm}$. A zona de inibição de crescimento para a F-OHf frente ao isolado de CgCV10 foi $31 \mathrm{~mm}$ em média. Frente a CgCV6 esse valor foi $27,5 \mathrm{~mm}$. Quando 0 isolado de CgCV5 foi submetido ao tratamento com a F-OHf a zona de inibição de crescimento foi em média $26,5 \mathrm{~mm}$. O isolado que apresentou menor média $(25,5 \mathrm{~mm})$ da zona de inibição de crescimento foi CgCV4. O FCZ não permitiu o crescimento em uma zona de $2,5 \mathrm{~mm}$ em média para três isolados, $\mathrm{CgCV} 5$, CgCV4, CgCV6. Apenas para um isolado a média de zona de inibição foi $1 \mathrm{~mm}$ para $\mathrm{FCZ}$, para o isolado CgCV10 (Figura 3). 


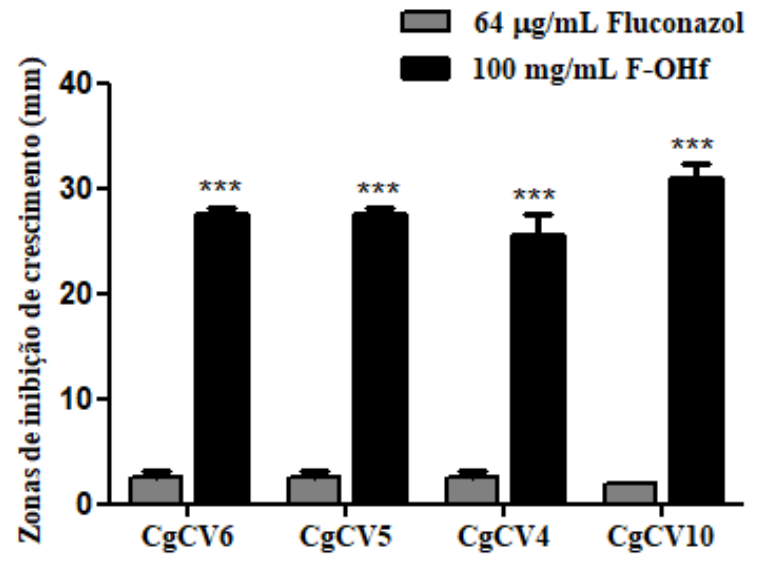

Isolados de $C$. glabrata

Figura 3. Zonas de inibição de crescimento $(\mathrm{ZIC})$ em milímetros $(\mathrm{mm})$ após o tratamento dos isolados clínicos de C. grablata com a fração de $n$-butanol a $100 \mathrm{mg} / \mathrm{mL}$ (F-OHf) e fluconazol $64 \mu \mathrm{g} / \mathrm{mL}$ (FCZ). O tratamento realizado com a F-OHf foi estatisticamente significativo comparado com controle realizado com FCZ para todos os isolados $\left({ }^{\star * *} p<0,001\right)$ considerando $95 \%$ de confiança utilizando o testes ANOVA e comparando os grupos como o teste de múltiplas comparações de Bonferroni.
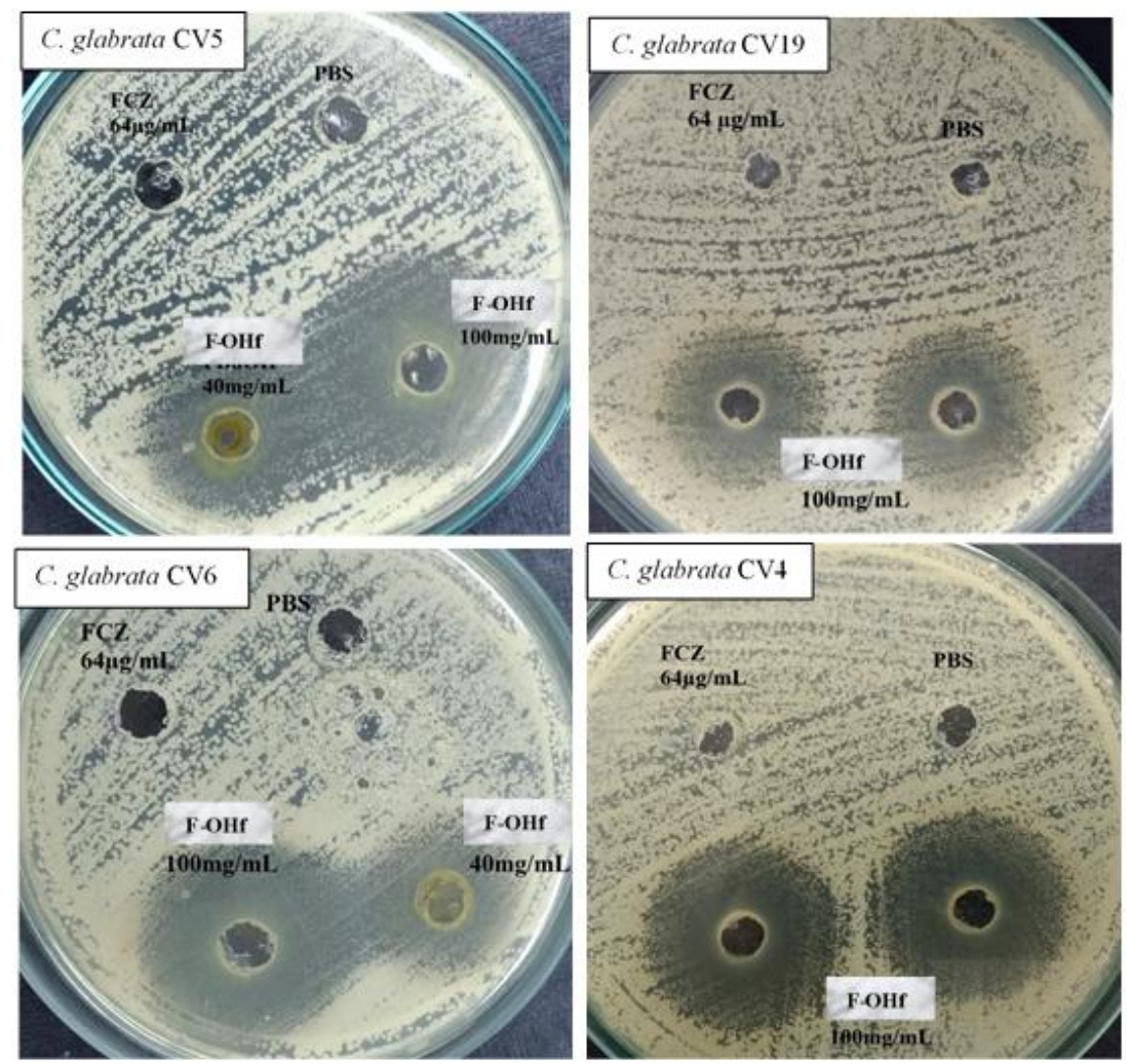

Figura 2. Avaliação da susceptibilidade da fração de $n$-butanol (F-OHf) por meio de difusão em ágar contra isolados CgCV10 (A), CgCV4 (B), CgCV6 (C) e CgCV5 (D) de secreção vaginal. Concentrações usadas: $40 \mathrm{mg} / \mathrm{mL}$ e $100 \mathrm{mg} / \mathrm{mL}$. Fluconazol $64 \mu \mathrm{g} / \mathrm{mL}$ e PBS $1 X$ foram usados como controles positivo e negativo, respectivamente. 
O tratamento com a $\mathrm{F}-\mathrm{OHf}$ na concentração de $100 \mathrm{mg} / \mathrm{mL}$ (figura 3) para todos os isolados foi significativo em relação ao controle realizado com FCZ $(p<0,001)$, porém não houve diferença estatística comparando o mesmo tratamento em isolados diferentes, tanto com FCZ quanto para a F-OHf. O PBS como já mencionado foi utilizado como controle negativo, portanto este não possibilitou a formação de zonas de inibição de crescimento para o isolado CgCV6 Apenas para um isolado a média de zona de inibição foi $1 \mathrm{~mm}$ para $\mathrm{FCZ}$, o isolado $\mathrm{CgCV} 10$ (Figura 3).

De acordo com os pontos de corte para a interpretação do teste de sensibilidades antifúngica ${ }^{24}$, os isolados de $C$. glabrata foram resistentes ao tratamento com o antifúngico FCZ. De forma semelhante, sob o ponto de vista de atividade inibitória, ocorreu formação de zonas de inibição de crescimento quando os isolados de C. glabrata foram tratamentos com F-OHf na concentração de $40 \mathrm{mg} / \mathrm{mL}$, esta promoveu a formação de zonas de inibição de crescimento em média de $29 \mathrm{~mm}$ contra $C$. glabrata CGCV5 Para o isolado de C. glabrata CGCV4 a F-FOH na concentração $40 \mathrm{mg} / \mathrm{mL}$ apresentou uma zona de inibição de crescimento igual a $25 \mathrm{~mm}$ em média. Contra os isolados de $C$. glabrata CGCV10 e C. glabrata F.J.F a zona de inibição formada por F-FOH foi em média de $21 \mathrm{~mm}$. O FCZ não permitiu o crescimento em uma zona de 2,5 $\mathrm{mm}$ em média para três isolados, $C$. glabrata CGCV5, C. glabrata CGCV4, C. glabrata



Figura 4. Zonas de inibição de crescimento $(\mathrm{ZIC})$ em milímetros $(\mathrm{mm})$ após o tratamento dos isolados clínicos de $C$. grablata com a fração de $n$-butanol a $40 \mathrm{mg} / \mathrm{mL}$ (F-OHf) e fluconazol $64 \mu \mathrm{g} / \mathrm{mL}$ (FCZ). O tratamento realizado com a F-OHf foi estatisticamente significativo comparado com controle realizado com FCZ para todos os isolados $\left({ }^{* *} p<0,001\right)$ considerando $95 \%$ de confiança utilizando o teste ANOVA e comparando os grupos como o teste de múltiplas comparações de Bonferroni. 
O tratamento com a $\mathrm{F}-\mathrm{OHf}$ contra todos os isolados de $C$. glabrata foi significativo em comparação com o controle realizado com fluconazol $(p<0,001)$. Comparando entre si o tratamento da $\mathrm{F}-\mathrm{OHf}$ contra os diferentes isolados, não houve resultado estatisticamente significativo ( $p>0,05)$.

\section{Discussão}

As leveduras do gênero Candida causam diferentes infecções em todo o mundo, especialmente em pacientes imunocomprometidos ${ }^{25}$, o fluconazol é o antifúngico de primeira escolha para o tratamento destas infecções mas cada vez mais é documentado o aumento de isolados cada vez mais resistentes a este fármaco ${ }^{18}$. No presente trabalho foi encontrado isolados da espécie $C$. glabrata resistentes ao fluconazol através do método ágar difusão, demonstrado na literatura como o padrão ouro para estabelecer a sensibilidade antifúngica destas leveduras ${ }^{26}$. Estes resultados mostram que em comparação ao antifúngico de primeira escolha a atividade inibitória de F-OHf foi melhor, utilizando esta técnica de difusão em ágar, em um trabalho anterior ${ }^{27}$, da mesma forma a fração butanólica obtida do extrato hidroalcólico de T. catappa apresentou melhor atividade nos isolados da espécie $C$. glabrata testados.

Vários trabalhos reportam a atividade de $T$. catappa contra vários microrganismos ${ }^{28,29,30}$, sendo escassos estudos com leveduras do gênero Candida, principalmente da espécie $C$. glabrata.

Em estudo realizado por Rocha ${ }^{31}$ os resultados obtidos corroboram com os do presente trabalho. Em seu trabalho foram utilizados isolados de Cryptococcus resistentes ao fluconazol. Os halos de inibição foram de até $28 \mathrm{~mm}$, demonstrando assim a sensibilidade destes isolados à fração butanólica de seu trabalho, validando ainda mais os resultados obtidos aqui.

Em um estudo também realizado com fração $n$-butanol de Terminalia catappa contra isolados de Candida provenientes de pacientes com câncer orogástrico ${ }^{20}$, foi observado que 0 extrato exerceu maior ação inibitória que o fluconazol, com halos de 23,6 $\mathrm{mm}$ enquanto que para o fluconazol o maior valor encontrado foi menor que $20 \mathrm{~mm}$, nenhum isolado utilizado no estudo era da espécie $C$. glabrata confirmando 0 pioneirismo deste trabalho.

A Terminalia catappa apresenta atividade antimicrobiana significativa contra vários microrganismos, como exemplo em um trabalho com Staphylococcus aureus e Pseudomonas aeruginosa ${ }^{32}$, evidenciando que esta planta tem atividade antimicrobiana significativa.

\section{Conclusão}

O uso da Terminalia catappa pode ser uma alternativa promissora para o tratamento da candidíase, estes resultados representam novas perspectivas para futuras pesquisas em continuação a este estudo para desenvolver terapias alternativas no combate destas infecções.

Em sua totalidade, os resultados obtidos neste trabalho demonstram 0 imenso potencial antifúngico da fração $n$-butanol das folhas de Terminalia catappa contra Candida glabrata. 


\section{Referências}

1. Barbosa LV, Mendes INFV, Alves MB, Campelo BC, Moura KCF, Nogueira NMC, Monteiro CA. Análise in silico do DNA genômico de três espécies do gênero Candida para verificação de ocorrência de microssatélites e observação de semelhanças interespecíficas. Revista de investigação biomédica. 2017; 9:6-16.

2. Alves $\mathrm{MB}$, Silva $\mathrm{IMO}$, Santos $\mathrm{Cl}$, França YR, Oliveira SKR, Monteiro SG, Monteiro CA. Prevalência de Candida spp. em amostras de secreção vaginal e sua relação com fatores associados à vulvovaginite. Revista de investigação biomédica.2015; 7:58-68.

3. Ragunathan L, Poongothai GK, Sinazer AR, Kannaiyan K, Gurumurthy H, Jaget N, Kuthalaramalingam S. Phenotypic Characterization and Antifungal Susceptibility Pattern to Fluconazole in Candida species Isolated from Vulvovaginal Candidiasis in a Tertiary Care Hospital. Journal of clinical and diagnostic research. 2014; 8(5).

4. Whaley SG, Berkow EL, Rybak JM, Nishimoto AT, Barker KS and Rogers PD. Azole Antifungal Resistance in Candida albicans and Emerging Non-albicans Candida Species. Front. Microbiol. 2017; 7:2173.

5. Furlaneto MC, Góes HP, Perini HF, Santos RC, Maia LF. How much do we know about hemolytic capability of pathogenic Candida species? 2018.

6. Arendrup, S; Sulim, A; Holm, L.; Nielsen, SD; Nielsen, JD; Knudsen, NE; Drenck, JJ; Christensen, HK; Johansen Questões de diagnóstico, características clínicas e resultados para os pacientes com fungemia. J. Clin. Microbiol., 2011;49 9:3300-3308

7. Costa CR. Fatores de virulência de isolados de Candida de pacientes imunocomprometidos. Caracterização molecular de C. albicans susceptíveis e resistentes ao fluconazol. Goiás [Tese de doutorado em Medicina Tropical - UFG Instituto de Patologia Tropical e Saúde Pública], 2009.
8. Guinea, J. Global trends in the distribution of Candida species causing candidemia. Clin. Microbiol. Infect. 2014, 20, 5-10.

9. Doi, AM, Pignatari ACC, Edmond MB, Marra AR, Camargo LFA, Siqueira RA et al. Epidemiology and microbiologic characterization of nosocomial candidemia from a Brazilian national surveillance program. Plos one, 2016,11(1)1-9

10. Matta DA, Souza ACR, Colombo AL. Revisiting Species Distribution and Antifungal Susceptibility of Candida Bloodstream Isolates from Latin American Medical Centers. 2017; 3-24.

11. Branco PVGC, Anjos DCV, Nascimento FB, Vale INF, Azevedo CMP, Monteiro SG, Figueiredo PMS, Monteiro CA. Prevalência e Produção de exoenzimas por espécies de Candida provenientes da mucosa bucal de pacientes com AIDS e indivíduos hígidos. Rev. Patol. Tropical. 2012; 41 (4).

12. Pfaller MA, Rhomberg PR, Messer, SA, Jones RN, Castanheira $M$. Isavuconazole, micafungin, and 8 comparator antifungal agents' susceptibility profiles for common and uncommon opportunistic fungi collected in 2013: temporal analysis of antifungal drug resistance using CLSI species-specific clinical breakpoints and proposed epidemiological cutoff values. Diagnostic Microbiology and Infectious Disease. 2015; 82: 303-13.

13. Pierce CG, Lopez-Ribot JL. Candidiasis drug discovery and development: new approaches targeting virulence for discovering and identifying new drugs. Expert Opinion on Drug Discovery. 2013; 8(9): 1117-1126.

14. Lortholary O, Desnos-Ollivier M, Sitbon $\mathrm{K}$, Fontanet A, Bretagne S, Dromer F. Recent exposure to caspofungin or fluconazole influences the epidemiology of candidemia: a prospective multicenter study involving 2,441 patients. Antimicrobial Agents and Chemotherapy. 2011; 55: 532-538.

15. Fothergill, A. W., Sutton, D. A., McCarthy, D. I., and Wiederhold, N. P. (2014). Impact of new antifungal breakpoints on antifungal resistance in Candida species. J. Clin. Microbiol. 52 
16. Oxman DA, Chow JK, Frendl G, Hadley $S$, Hershkovitz $S$, Ireland $P$, et al. Candidaemia associated with decreased in vitro fluconazole susceptibility: is Candida speciation predictive of the susceptibility pattern? J. Antimicrob. Chemother. 2010; 65: 1460-1465.

17. Pfaller MA. Antifungal drug resistance: mechanisms, epidemiology, and consequences for treatment. The American Journal of Medicine. 2012;125: S3-S13.

18. Cui, J.; Ren, B.; Tong, Y.; Dai, H.; Zhang, L. Synergistic combinations of antifungals and anti-virulence agents to fight against Candida albicans. Virulence. 2015; 6(4): 362-371.

19. Rocha FMG. Avaliação da combinação in vitro entre o extrato e a fração butanólica de Terminalia catappa e fluconazol frente a Candida albicans isolados de pacientes com câncer orogástrico [Monografia]. São Luís: Instituto Federal de educação, ciência e tecnologia do Maranhão; 2017.

20. Terças AL, Marques SG, Moffa EB, Alves MB, Azevedo CM, Siqueira W L, Monteiro CA. Antifungal Drug Susceptibility of Candida Species Isolated from HIVPositive Patients Recruited at a Public Hospital in São Luís, Maranhão, Brazil. Frontiers in microbiology. 2017; 8298.

21. Anand, A. V.; Divya, N.; Kotti, P. P. An updated review of Terminalia catappa. Pharmacognosy Reviews. 2015; 9: 9398.

22. Mininel FJ, Leonardo Junior CS, Espanha $L G$, Resende FA, Varanda EA, Leite $C Q$, et al. Characterization and quantification of compounds in the hydroalcoholic extract of the leaves from Terminalia catappa Linn. (Combretaceae) and their mutagenic activity. Evid Based Complement Alternat Med. 2014.

23. Clinical And Laboratory Standards Institute. Reference method for broth dilution antifungal susceptibility testing of yeasts. Approved standard M27A3, 3rd ed. Clinical and Laboratory Standards Institute, Wayne, PA. 2008.

24. Pour HA, Salari S, Almani PGN. Oropharyngeal candidiasis in HIV/AIDS patients and non-HIV subjects in the
Southeast of Iran. Current medical mycology. 2018; 4(4): 1-6.

25. Li, X.; Hou, Y.; Yue, L.; Liu, S.; Du, J.; Sun, S. Potential targets for antifungal drug discovery based on growth and virulence in Candida albicans. Antimicrobial Agents and Chemotherapy. 2015; 59: 5885-5891, 2015.

26. Terças, A. G., et al. Phytochemical characterization of Terminalia catappa Linn. extracts and their antifungal activities against Candida spp. Front Microbiol. 2017; 8: 595.

27. Akharaiyi, F.C.; Ilori, R.M.; Adesida, J.A. Antibacterial effect of Terminalia catappa on some selected pathogenic bacteria. International Journal of Pharmaceutical and Biomedical Research, v. 2, p. 64-7, 2011. Mandloi S, Mishra R, Varma R, Varughese B, Tripathi J. A study on phytochemical and antifungal activity of leaf extracts of Terminalia cattapa. International Journal of Pharma \& Bio Sciences. 2013; 4: B1385-B1393.

28. Taganna, J.C., Quanico, J.P., Perono, R.M., Amor, C.E., Rivera, W.L. Tanninrich fraction from Terminalia catappa inhibits quorum sensing (QS) in Chromobacterium violaceum and the QScontrolled biofilm maturation and LasA staphylolytic activity in Pseudomonas aeruginosa. Journal of Ethnopharmacology. 2011; 134:p. 865871.

29. Rocha CHL. Atividade antifúngica e antivirulência da fração butanólica do extrato de Terminalia catappa em combinação com fluconazol contra isolados de Cryptococcus gattii [Monografia]. São Luís: Instituto Federal de educação, ciência e tecnologia do Maranhão; 2017.

30. Allyn $O Q$, Kusumawati $E$, Nugroho $R$ A. Antimicrobial activity of Terminalia catappa brown leaf extracts against Staphylococcus aureus ATCC 25923 and Pseudomonas aeruginosa ATCC27853. F1000 Research. 2018. 